\title{
Estimation des flux particulaires dans les canaux d'amenée des barrages-réservoirs Seine et Aube, analyse méthodologique comparative
}

\section{Evaluation of sediment discharge passing through inflow channels of Seine and Aube reservoirs, comparison of methodic analysis}

\author{
C. Martin ${ }^{(1)}$ et I. Teulières (2) \\ (1) Institut des Sciences et Techniques de l'Environnement - Laboratoire d'Hydrobiologie - \\ Hydroécologie. Place Leclerc, 25030 Besançon - Cedex. \\ (2) Institution Interdépartementale des Barrages-Réservoirs du Bassin de la Seine - 8, rue Vil- \\ liot - 75012 Paris.
}

\begin{abstract}
Résumé. - Dans une perspective de maîtrise de la qualité des eaux, stockées durant les crues, et restituées à l'étiage des grands cours d'eau, l'Institution Interdépartementale des Barrages-Réservoirs du Bassin de la Seine (I.I.B.R.B.S.), s'intéresse depuis 1990, dans le cadre d'un programme pluriannuel, à la quantification des flux particulaires.

Le protocole original, conçu pour l'estimation est présenté. Les résultats obtenus par les collecteurs, décrits dans cet article, sont confrontés aux analyses des sédiments des canaux d'amenée, et aux flux particulaires évalués par le prélèvement hebdomadaire des matières en suspension.

On présente les premières estimations des quantités de matière particulaire (MES, COP, Nk, Ptot.) dérivées annuellement dans les réservoirs. L'influence relative des divers épisodes de prise est discutée.
\end{abstract}

Mots clés. - gestion écologique, lacs de barrages, canaux, matières en suspension, flux.

Abstract. - In order to control the quality of the water filling up the storage basins and released to the rivers at low water level, the Institution Interdépartementale des Barrages-Réservoirs du Bassin de la Seine (I.I.B.R.B.S.) is developing a survey program in which sediment discharge is studied since 1990.

Estimation methodology developed is presented. Results obtained from original sediment 
traps are compared with sediment analysis values and with sediment discharge measured by collecting suspended matter weekly.

First estimations concerning Suspended Matter, Particulate Organic Carbon, Particulate Kjeldahl Nitrogen, and Particulate Total Phosphorus inflows (tons by year), are presented. Relative effects of seasonally filling up periods are discussed.

Key words. - Lake management, Storage basin, Channels, Suspended Matter, Particulate fluxes.

\section{INTRODUCTION}

Les lacs, naturels ou artificiels, se comportent comme de véritables "pièges à substances" (Dussart 1966), dont l'évolution naturelle tend au comblement. La sénescence du lac et l'augmentation de la fraction organique, s'associent à ce processus (Martin 1993).

$\mathrm{Si}$, la géochimie des eaux est essentielle dans les processus de transport des constituants (Meybeck 1983, Ertel \& al. 1986), l'intérêt de l'approche de la fraction particulaire est montrée par tous les travaux, peu nombreux, qui l'ont abordée (Hedges \& al. 1986, Fatorelli \& al. 1988, Stabel \& Geiger 1985). Associés aux particules en suspension, les apports en constituants organiques (carbone, azote, phosphore), contribuent à orienter le mode de fonctionnement lacustre dont dépend la qualité et l'évolution trophique du système (Golterman 1973, Jaquet 1985).

La détermination de la composition des matières particulaires pose le problème de quantité de matière disponible à l'analyse. A moins que ne soient retenus des procédés adaptés aux microquantités (Van Put \& al. 1994), I'utilisation de techniques analytiques classiques nécessitent géné- ralement des quantités de matière sèche, qui ne peuvent être obtenues que par un échantillonnage de plusieurs dizaines de litres d'eau brute ou par l'utilisation de centrifugeuses à flux continu de gros débit (Golterman \& al. 1983).

Les contraintes de ce mode de prélèvement conduisent certains auteurs à équiper les sites d'étude de stations expérimentales (Fattorelli \& al. op. cit.), ou à installer des collecteurs à sédiment en recherchant les corrélations avec d'autres méthodes (Favager \& al. 1991).

Dans le cadre d'un programme pluridisciplinaire, pluriannuel de contrôle et de maîtrise des eaux prélevées et restituées, l'Institution Interdépartementale des Barrages-Réservoirs du Bassin de la Seine (I.I.B.R.B.S.), s'intéresse depuis 1990 à la quantification des flux particulaires entrant dans les réservoirs, en expérimentant la méthode des collecteurs à sédiments.

\section{MATÉRIEL ET MÉTHODES}

\subsection{Les lacs}

Les Barrages-Réservoirs situés à l'est de Paris en dérivation de la Marne (lac du Der), de la Seine (lac 
d'Orient) et de l'Aube (lacs AmancePort Dienville et du Temple) représentent une capacité de stockage de plus de $700 \mathrm{Mm}^{3}$ pour des débits maximum dérivés, variant de 135 à $400 \mathrm{~m}^{3} / \mathrm{s}$. Viennent s'y ajouter trois barrages en front de vallée, dont deux font l'objet d'une gestion commune avec Electricité De France (fig. 1).

Les réservoirs Seine et Aube, objets de notre étude, sont alimentés, pour le premier par les eaux de la

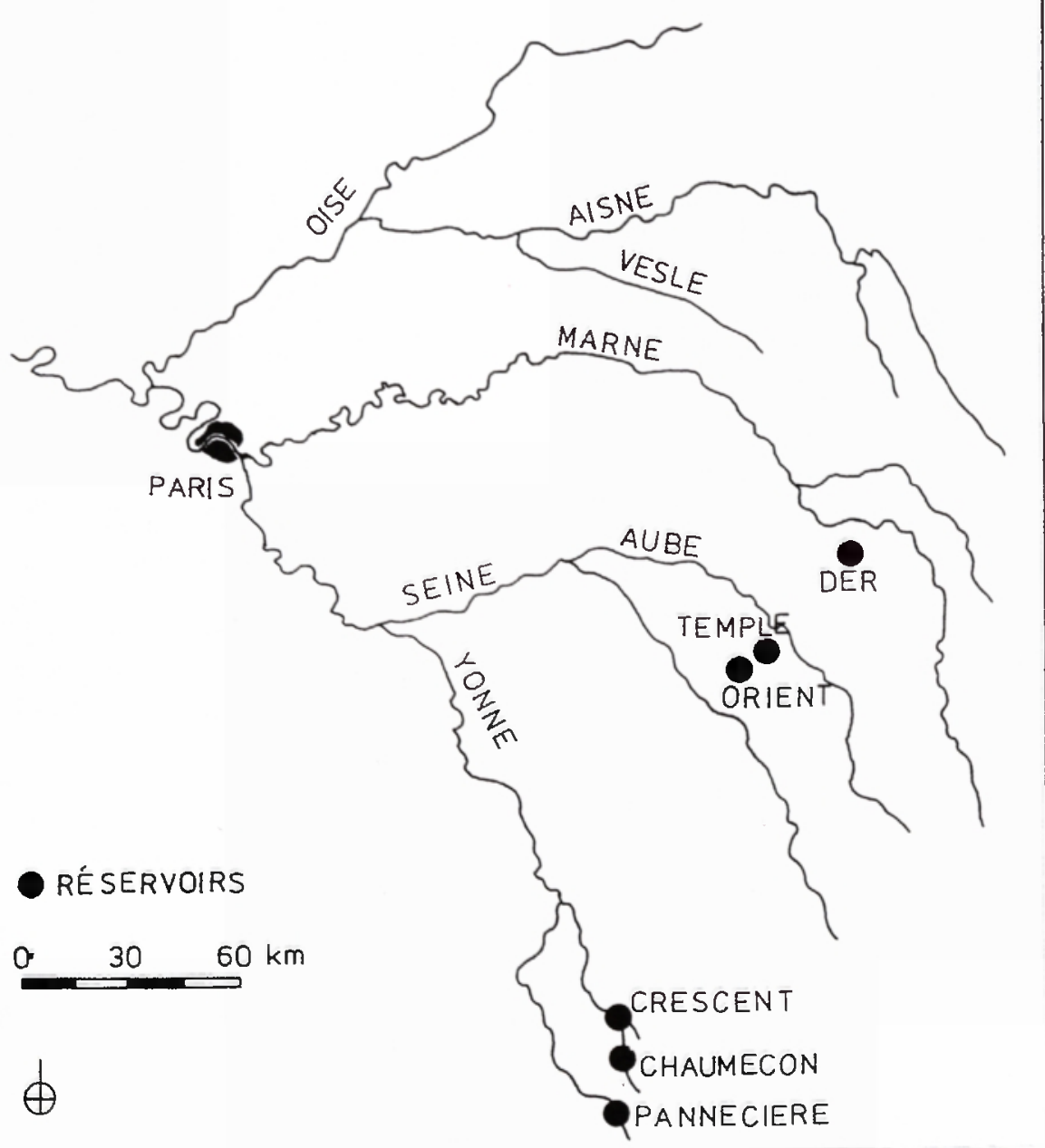

Fig. 1. - Localisation des Barrages-réservoirs sur le bassin hydrographique de la Seine. Fig. 1. - Location of storage basins in the drainage area of the Seine river. 
Seine par un canal d'amenée d'environ $13 \mathrm{~km}$, et par les eaux de l'Aube pour le second dont le canal d'amenée mesure environ $3,8 \mathrm{~km}$.

Au cours des deux années d'étude, les réservoirs ont été remplis en trois prises principales: décembre, février, avril (figs. 2 et 3 ). Les débits maximums enregistrés sont modérés au vu des possibilités d'écrêtement (tableau I)

\subsection{Echantillonnage et analyses}

Les sédiments déposés dans les canaux d'amenée sont récoltés à l'occasion d'une mise à sec en 1990. Les échantillons soumis à l'analyse résultent de l'homogénéisation d'une dizaine de sous-échantillons d'un $\mathrm{dm}^{3}$ effectués au préleveur à main. Sur le canal Aube, les prélèvements sont réalisés $1 \mathrm{~km}$. environ à l'aval de la prise d'eau, et à quelques dizaines de mètres à l'amont du déversoir. Sur le canal Seine un premier prélèvement est situé à environ $4 \mathrm{~km}$ de la prise d'eau, un second $6 \mathrm{~km}$ à l'aval, et un troisième à quelques dizaines de mètres à l'amont du vannage d'extrémité.

Le tonnage de sédiments déposés est évalué sur le canal Seine après un relevé systématique des épaisseurs sur la longueur totale du canal. La conversion des épaisseurs, en tonnage de matière sèche est effectuée par tronçon, en tenant compte de la teneur en eau, et d'une densité des

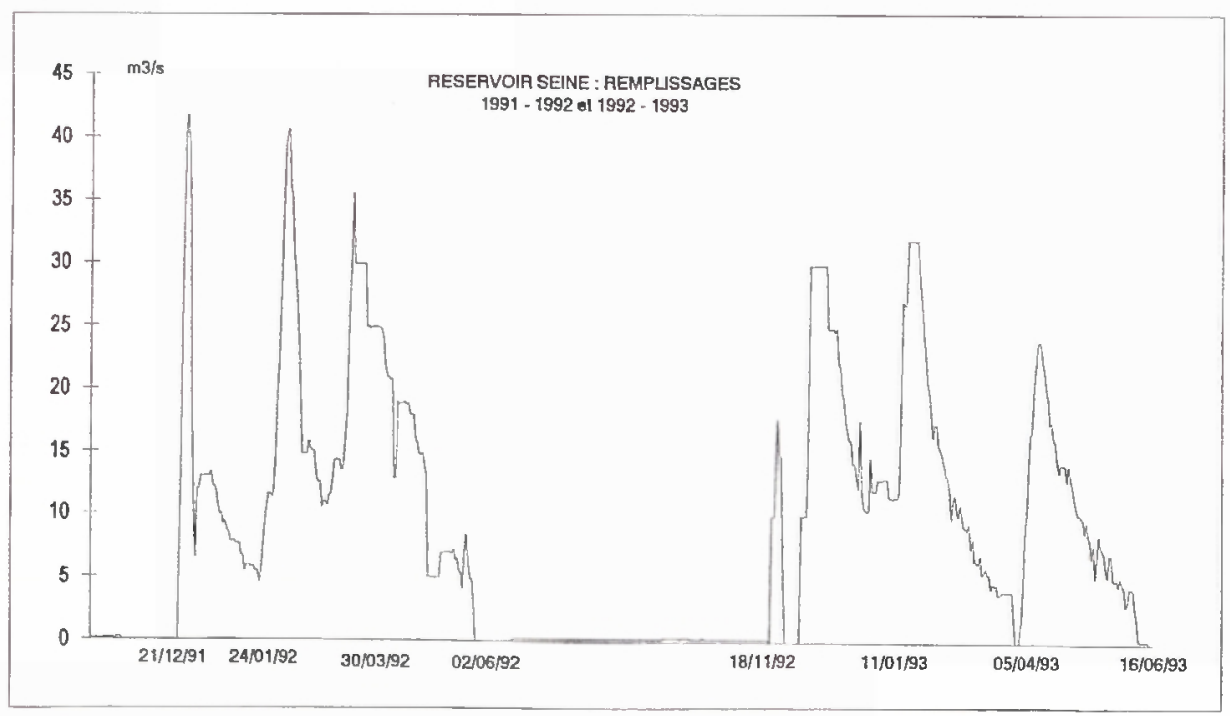

Fig. 2. - Évolution des débits de prise au cours des deux cycles de remplissages. - 1991 - 1992 et 1992 - 1993. Barrage-réservoir Seine.

Fig. 2. - Water flow values of two hydrological cycles of filling up the Seine reservoir, 1991 1992, $1992-1993$. 


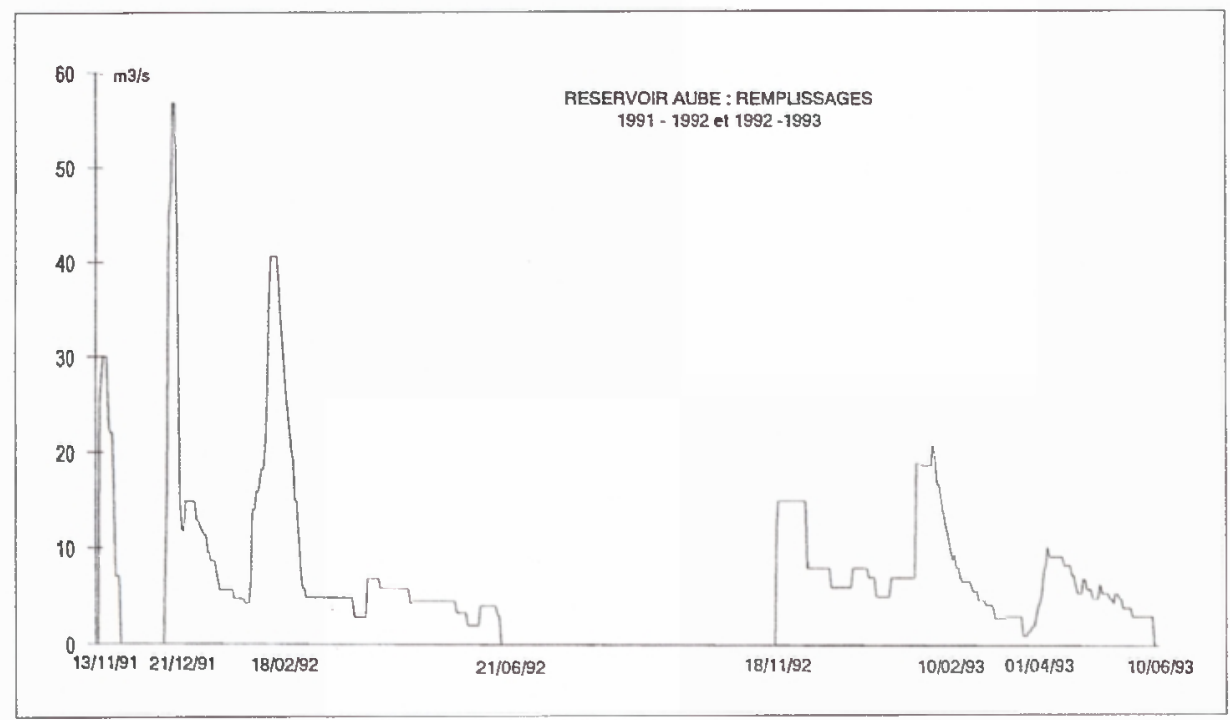

Fig. 3. - Évolution des débits de prise au cours des deux cycles de remplissages. - 1991 - 1992 et 1992 - 1993. Barrage-réservoir Aube.

Fig. 3. - Water flow values of two hydrological cycles of filling up the Aube reservoir, $1991-1992$, 1992 - 1993.

Tableau I. - Caractéristiques géomorphologiques et hydrauliques générales des barrages-réservoirs Seine et Aube.

Table I. - General geomorphological and hydrological features of Seine et Aube reservoirs.

\begin{tabular}{|l|c|c|}
\hline & Réservoir Seine & Réservoir Aube \\
\hline $\begin{array}{l}\text { bassin versant contrôlé } \\
\left(\mathrm{km}^{2}\right)\end{array}$ & 2300 & 1550 \\
\hline $\begin{array}{l}\text { superficie de la retenue } \\
\left(\mathrm{km}^{2}\right)\end{array}$ & 23,5 & 25 \\
\hline capacité totale (Mm3) & 205 & 170 \\
\hline année de mise en service & 1966 & 1990 \\
\hline $\begin{array}{l}\text { débit maximum } \\
\text { d'écrêtement (m3/s) }\end{array}$ & 180 & 135 \\
\hline $\begin{array}{l}\text { débit maximum de soutién } \\
\text { d'étiage (m3/s) }\end{array}$ & 35 & 35 \\
\hline
\end{tabular}


sédiments secs approximée à $2,65 \mathrm{~g} / \mathrm{cm}^{3}$ (Bloesch \& al. 1982, Hakanson et Jansson 1983, Bapst 1987). Le taux de sédimentation annuel est obtenu en divisant les tonnages estimés par les vingt-quatre années de fonctionnement du canal.

Entre le 23 novembre 1991 et le 14 avril 1992, le taux de sédimentation annuel dans le canal Seine est estimé par la pose de collecteurs, conçus et réalisés aux ateliers de la circonscription Aube - Seine - Yonne.

Le système préleveur est constitué d'une partie lestée et protectrice destinée à lutter contre le courant et d'un collecteur en P.V.C. (hauteur $=60 \mathrm{~cm}$, diamètre $=24 \mathrm{~cm}$ ) dans lequel est adapté une structure alvéolaire permettant d'atténuer les turbulences internes tout en conservant une surface de réception élevée $\left(0,05 \mathrm{~m}^{2}\right)$. L'ensemble (150 à $200 \mathrm{~kg}$ ) est treuillé à partir d'un engin tracteur (fig. 4). La fréquence des levées est d'environ trois semaines.

Entre le 8 janvier et le 22 juin 1993, les collecteurs sont installés dans les canaux Aube et Seine à quelques centaines de mètres à l'aval de la prise d'eau en vue de confronter les quantités de matière récoltées et le flux particulaire estimé par le dosage des matières en suspension sur un échantillon hebdomadaire prélevé sur le site d'étude.

Sur les sédiments et sur les suspensions recueillies dans les collecteurs, les échantillons sont séchés à $80^{\circ} \mathrm{C}$ jusqu'à poids constant, broyés et homogénéisés. Le carbone organi- que est dosé par la méthode Anne. L'azote est dosé par la méthode Kjeldahl (AFNOR EP118E). Le phosphore total est dosé par colorimétrie après attaque nitroperchlorique. La concentration en matière en suspension, est déterminée selon la méthode normalisée (Rodier 1984, norme AFNOR NFT 90-105, 1979), après filtration sur filtre Millipore AP-20 de 0,45 $\mu \mathrm{m}$ de diamètre de pores.

\section{RÉSULTATS}

\subsection{Composition des sédiments et des suspensions recueillies dans les collecteurs}

Les compositions moyennes en carbone organique, azote Kjeldahl et phosphore total des sédiments et des suspensions recueillies dans les collecteurs sont présentées au tableau II. Les amplitudes indiquent les variabilités spatiales et saisonnières des valeurs.

Les sédiments des canaux des deux réservoirs présentent des compositions similaires (Corg. : 2 $3 \%$ m.s., Nk : $0,4-0,5 \%$ m.s., Pt : $0,2-0,3 \%$ m.s.), qui témoignent d'une faible fraction organique.

Entre les sédiments et les suspensions, les écarts s'expriment essentiellement pour le carbone organique et l'azote Kjeldahl dont les plus fortes teneurs moyennes dans les suspensions (Corg. : $4-6 \%$ m.s., Nk : 0,5 - $0,8 \%$ m.s.) peuvent être rappro- 

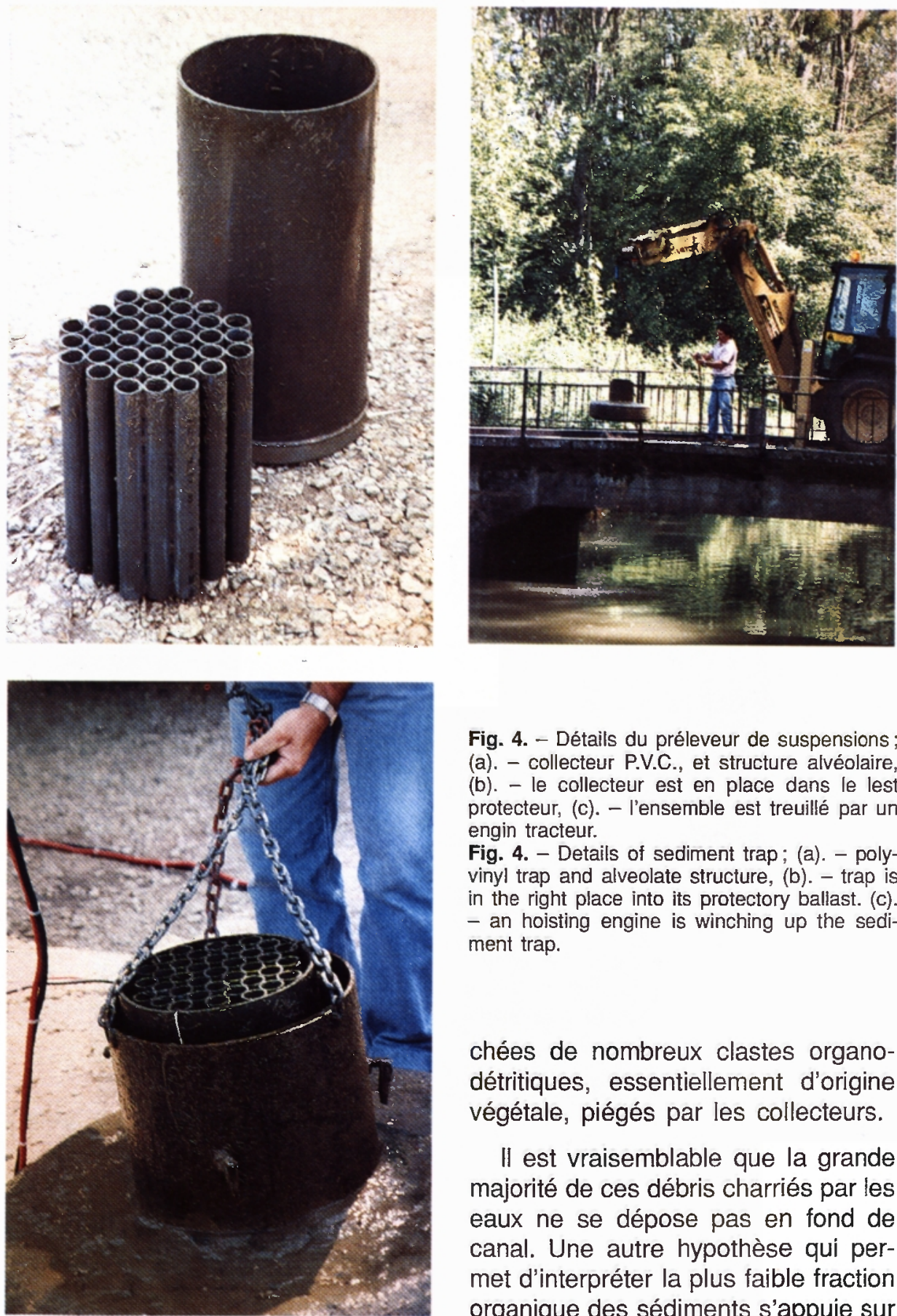

Fig. 4. - Détails du préleveur de suspensions; (a). - collecteur P.V.C., et structure alvéolaire, (b). - le collecteur est en place dans le lest protecteur, (c). - l'ensemble est treuillé par un engin tracteur.

Fig. 4. - Details of sediment trap; (a). - polyvinyl trap and alveolate structure, (b). - trap is in the right place into its protectory baliast. (c). - an hoisting engine is winching up the sediment trap.

chées de nombreux clastes organodétritiques, essentiellement d'origine végétale, piégés par les collecteurs.

II est vraisemblable que la grande majorité de ces débris charriés par les eaux ne se dépose pas en fond de canal. Une autre hypothèse qui permet d'interpréter la plus faible fraction organique des sédiments s'appuie sur 
Tableau II. - Composition moyenne et amplitudes de variation des valeurs en carbone organique, azote Kjeldahl et phosphore total dosés sur les sédiments prélevés dans les canaux et sur les suspensions recueillies dans les collecteurs. Teneurs exprimées en pour cent de matière sèche.

Table II. - Composition mean values and deviation values for organic carbon, Kjeldah! nitrogen and total phophorus from sediments of inflow channels and suspended matter catched by traps. Terms are expressed amount per cent of dry matter.

\begin{tabular}{|c|c|c|c|}
\hline \multicolumn{5}{|c|}{ SEINE } \\
\hline & sédiments & $\begin{array}{c}\text { suspensions } \\
1991-1992\end{array}$ & $\begin{array}{c}\text { suspensions } \\
1992-1993\end{array}$ \\
\hline carbone organique & $2,68 \pm 0,16$ & $4,60 \pm 0,90$ & $6,02 \pm 4,88$ \\
\hline azote Kjeldahl & $0,44 \pm 0,06$ & $0,52 \pm 0,10$ & $0,63 \pm 0,26$ \\
\hline phosphore total & $0,280 \pm 0,032$ & $0,129 \pm 0,110$ & $0,352 \pm 0,263$ \\
\hline \multicolumn{4}{|c|}{ AUBE } \\
\hline & sédiments & suspensions & suspensions \\
& $1991-1992$ & $1992-1993$ \\
\hline carbone organique & $2,15 \pm 0,55$ & $5,40 \pm 1,20$ & $5,33 \pm 2,27$ \\
\hline azote Keldahl & $0,43 \pm 0,11$ & $0,61 \pm 0,28$ & $0,78 \pm 0,36$ \\
\hline phosphore total & $0,215 \pm 0,015$ & $0,126 \pm 0,046$ & $0,262 \pm 0,173$ \\
\hline
\end{tabular}

la minéralisation progressive de la matière organique au cours de la période estivale durant laquelle les canaux sont non fonctionnels.

Les observations relatives au carbone et à l'azote ne concernent pas le phosphore total, dont les teneurs moyennes similaires dans les sédiments et les suspensions, suggèrent qu'il se présente essentiellement sous une forme minérale, aisément sédimentée et non biodisponible.

Au cours des deux cycles de remplissages étudiés, l'utilisation des collecteurs permet de mettre en évidence la variabilité interannuelle de la composition des suspensions. Les deux réservoirs situés à proximité géographique, sont cependant aljmentés par les eaux de deux bassins versants distincts. II est instructif de noter que la qualité des apports particulaires semble suivre pour les deux réservoirs une dynamique interannuelle similaire. On peut considérer que les teneurs moyennes en azote restent plus élevées sur Aube $(0,6-$ $0,8 \%$ m.s.) que sur Seine $(0,5-0,6 \%$ m.s.) et inversement, que les teneurs en phosphore total restent plus faibles sur Aube $(0,1-0,3 \%$ m.s.) que sur Seine $(0,1-0,4 \%$ m.s. $)$.

\subsection{Taux de sédimentation dans les canaux}

Les données recueillies sur les sédiments du canal Seine en 1990 permettent d'estimer à 3975 tonnes, la quantité de matière sèche déposée 
dans le canal entre 1966 et 1990 , soit une sédimentation moyenne de 165 tonnes/an. Selon cette estimation et sur la base des compositions moyennes, les taux de sédimentation annuels des constituants analysés sont évalués et comparés aux résultats obtenus par les collecteurs (tableau III).

Compte-tenu de la méthodologie développée et des approximations effectuées, seuls les ordres de grandeurs peuvent être retenus, et témoignent d'une bonne concordance des valeurs à l'exception du carbone organique particulaire.

L'écart observé sur les taux de carbone organique (4 tonnes/an selon l'estimation effectuée sur les sédiments, contre 14 tonnes/an pour les collecteurs), doit être rapproché des remarques effectuées précédemment et relatives à la nature des apports organiques. Les collecteurs paraissent surestimer les taux de sédimen- tation pour les constituants organiques de larges surfaces, aisément mobilisés aux vitesses de courant observées pouvant atteindre $1 \mathrm{~m} / \mathrm{s}$ (Fischer \& al. 1979).

En première analyse, et notamment en ce qui concerne le carbone organique particulaire, les compositions et les taux de sédimentation obtenus par les collecteurs sont davantage représentatifs de l'épisode hydrologique que des sédiments déposés. La comparaison des valeurs entre sédiments et collecteurs, montre une bonne concordance des ordres de grandeurs concernant la sédimentation en fond de canal du phosphore total et, dans une certaine mesure, de la matière sèche et de l'azote Kjeldahl.

\subsection{Flux particulaires}

La comparaison portera sur les flux captés par les collecteurs (grammes

Tableau III. - Taux de sédimentation annuelle de la matière sèche, du carbone organique particulaire, de l'azote Kjeldahl particulaire, et du phosphore total particulaire, estimés sur le canal Seine par les sédiments et les collecteurs.

Table III. - dry matter, particulate organic carbon, particulate Kjeldahl nitrogen and particulate total phophorus, sedimentation rates estimated for the Seine inflow channel, by sediments and traps methods.

\begin{tabular}{|c|c|c|}
\hline & sédiments & collecteurs \\
\hline $\begin{array}{c}\text { matière sèche } \\
\text { (tonnes/an) }\end{array}$ & 165,6 & 307,7 \\
\hline $\begin{array}{c}\text { carbone organique } \\
\text { particulaire (tonnes/an) }\end{array}$ & 4,44 & 14,12 \\
\hline $\begin{array}{c}\text { azote Kjeldahl } \\
\text { particulaire (tonnes/an) }\end{array}$ & 0,73 & 1,60 \\
\hline $\begin{array}{c}\text { phosphore total } \\
\text { particulaire (tonnes/an) }\end{array}$ & 0,46 & 0,40 \\
\hline
\end{tabular}


par jour) et les flux particulaires (tonnes par jour) évalués d'après les concentrations en m.e.s. Seule la matière sèche est étudiée, les dosages des constituants n'ayant été envisagés que sur les échantillons recueillis par les collecteurs.

Les figures 5 et 6 présentent les résultats obtenus pour les deux canaux, au cours des périodes de prises étudiées; la première en février, s'effectue avec des débits maximums de $30-40 \mathrm{~m}^{3} / \mathrm{s}$ pour le canal Seine et de $20-25 \mathrm{~m}^{3} / \mathrm{s}$ pour le canal Aube, la seconde au mois d'avril s'avère plus modérée avec des débits maximum de 20 à $30 \mathrm{~m}^{3} / \mathrm{s}$ sur le canal Seine et 10 à $15 \mathrm{~m}^{3} / \mathrm{s}$ sur le canal Aube.
Le flux particulaire le plus élevé approche les 80 tonnes/jour le 2 février 1993 sur le canal Seine. Sur les deux figures on observe une réduction des flux particulaires conjointes avec la réduction des prises. En période de débits modérés, les flux se maintiennent à moins de 5 tonnes/jour.

Sur le réservoir Aube, le 8 mai, une valeur de concentration se distingue et quitte l'ordre de grandeur général. Les relevés pluviométriques journaliers montrent une période d'orage au cours des jours précédents. L'épisode, de courte durée, n'a pas donné lieu à une variation des débits de prise, tout en entraînant une augmentation passagère mais intense de la turbidité des eaux.

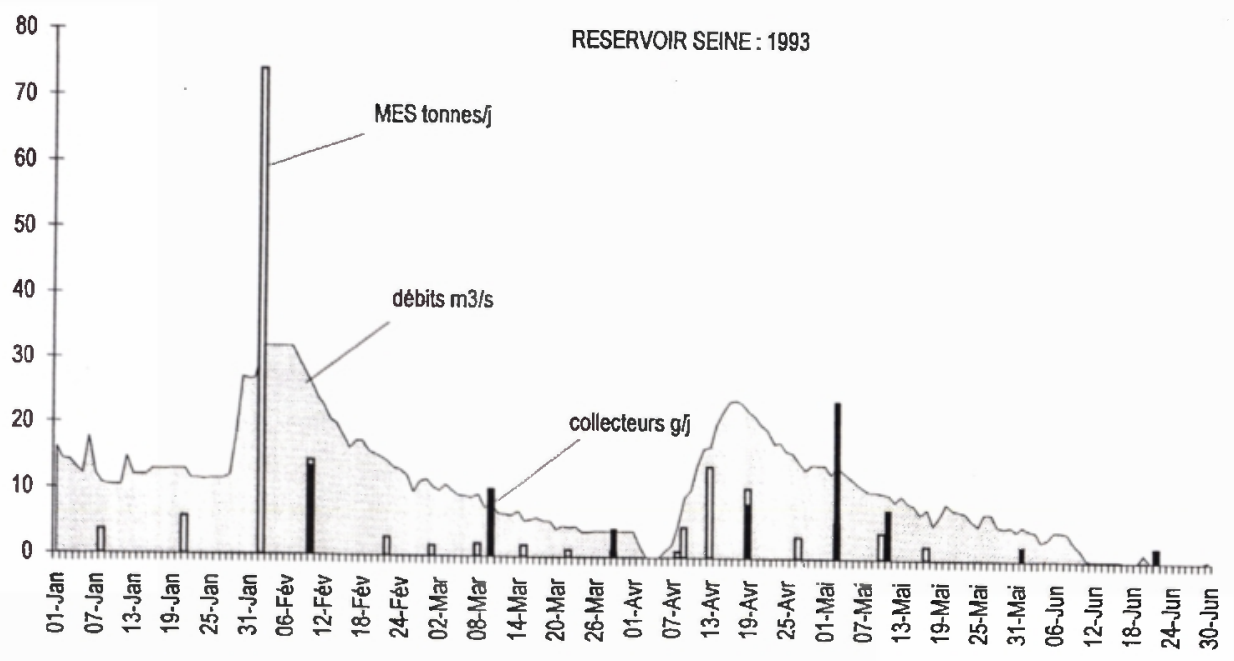

Fig. 5. - Représentation graphique des flux particulaires (MES. - tonnes/j) et des flux captés par les collecteurs $(\mathrm{g} / \mathrm{j})$, confrontés aux débits de prise $\left(\mathrm{m}^{3} / \mathrm{s}\right)$. Réservoir Seine, 1993.

Fig. 5. - Diagram of sediment discharge (Suspended Matter. - ton./d.) and settling fluxes measured by traps (g./d.), compared with water discharge $\left(\mathrm{m}^{3} / \mathrm{s}\right)$. Seine reservoir, 1993. 


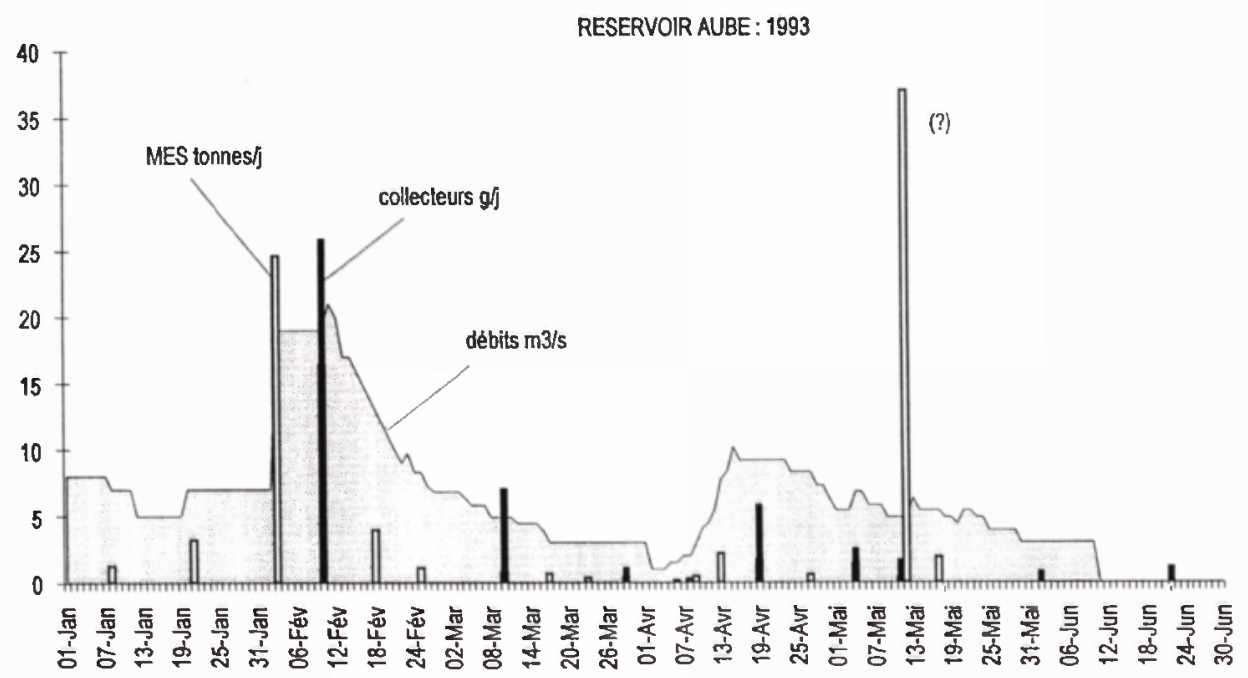

Fig. 6. - Représentation graphique des flux particulaires (MES. - tonnes/j) et des flux captés par les collecteurs $(\mathrm{g} / \mathrm{j})$, controntés aux débits de prise $\left(\mathrm{m}^{3} / \mathrm{s}\right)$. Réservoir Aube, 1993.

Fig. 6. - Diagram of sediment discharge (Suspended Matter. - ton./d.) and settling fluxes measured by traps (g./d.), compared with water discharge $\left(\mathrm{m}^{3} / \mathrm{s}\right)$. Aube reservoir, 1993.

Les valeurs brutes concernant les suspensions recueillies dans les collecteurs sont exprimées en grammes par jour. Elles sont représentées sur le graphique le jour de la levée et intègrent la totalité de la période qui court depuis la date de la précédente valeur.

Sur les deux canaux les valeurs maximums sont du même ordre de grandeur $(25-30 \mathrm{~g} / \mathrm{j})$; elles sont toutefois observées à des périodes différentes (en mai sur Seine, en février sur Aube). En dehors de ces deux pics, l'amplitude des valeurs est modérée et ces dernières se maintiennent généralement entre 10 et $20 \mathrm{~g} / \mathrm{j}$ sur Seine, et entre 2 et $10 \mathrm{~g} / \mathrm{j}$ sur Aube.
Les relations entre les trois composantes étudiées sont mises en évidence à la figure 7 . Les points correspondent aux huit périodes de fonctionnement des collecteurs. Pour chacune des périodes, les remplissages sont représentés par le débit moyen journalier $\left(\mathrm{Mm}^{3} / \mathrm{j}\right)$, les flux particulaires (MES : tonnes/jour) résultent de l'intégration des valeurs instantanées sur la période considérée, les valeurs des collecteurs $\mathrm{g} / \mathrm{j}$ sont celles des figures 5 et 6 .

Sur les deux réservoirs, on retrouve la loi générale de l'augmentation conjointe des débits «solides» et «liquides». La proportionnalité est respectée pour les débits inférieurs à $1 \mathrm{Mm}^{3} / \mathrm{j}$. Au-delà, les flux solides aug- 


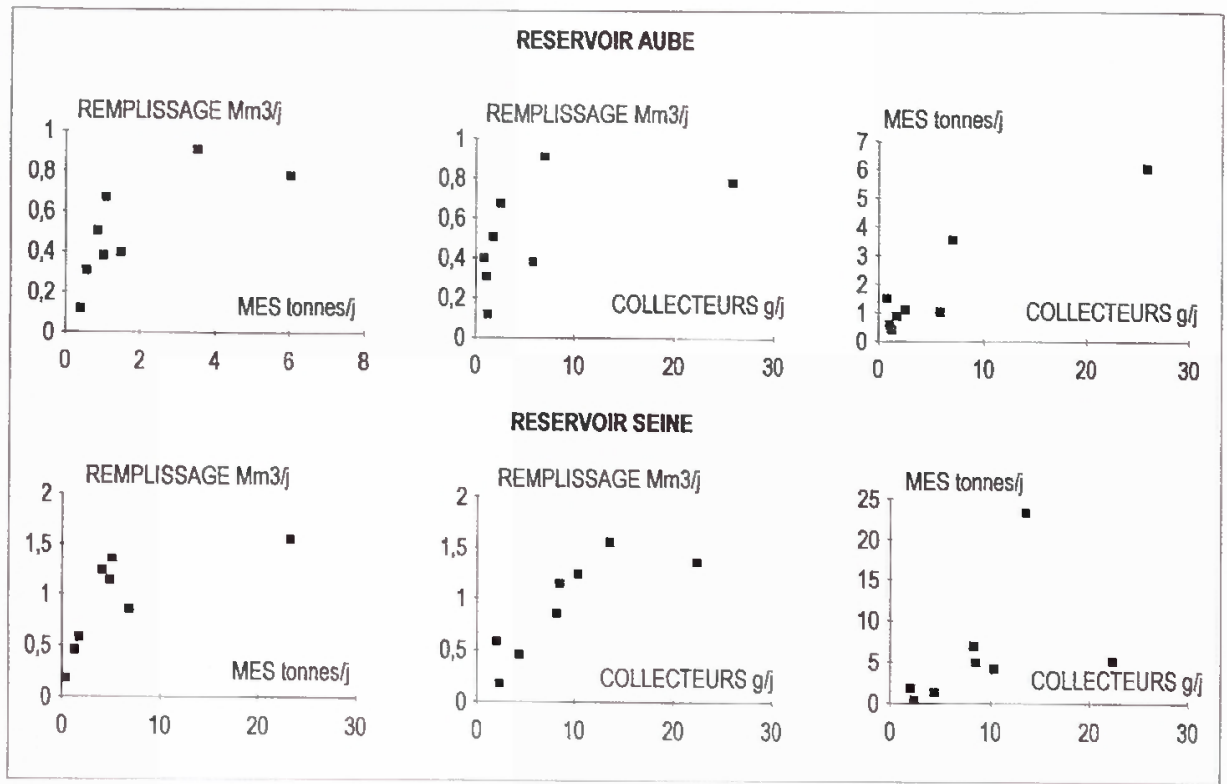

Fig. 7. - Graphiques $Y=f(X)$ établis pour les flux hydrauliques (remplissage $M m^{3} / \mathrm{j}$ ), les flux particulaires (tonnes/j) et les flux captés par les collecteurs $(g / j)$. Réservoirs Aube et Seine, 1993. Fig. 7. - Graphics $Y=f(X)$ plotted with water discharge $\left(\mathrm{Mm}^{3} / \mathrm{j}\right)$ ), sediment discharge (ton./d) and settling fluxes measured by traps (g./d.). Aube et Seine reservoirs, 1993.

mentent plus rapidement que les flux hydrauliques, selon une régression générale du type exponentielle (Meybeck, 1985) dont les constantes restent à définir.

A terme, les régressions établies entre les trois composantes constitueront pour une station donnée, la calibration des résultats des collecteurs. Il sera nécessaire de valider ces courbes par des mesures effectuées dans des gammes de débits plus étendues que celles observées ici.

En première approximation, et malgré une dispersion des points sur Seine, la relation entre flux particulaires et flux captés par les collec- teurs, est acceptée comme linéaire pour le calcul des premières estimations discutées ci-après.

\section{DISCUSSION}

Le tableau IV présente les premières estimations des flux particulaires dérivés annuellement lors des remplissages et calculés à partir des prélèvements hebdomadaires de MES et des analyses effectuées sur les échantillons récoltés dans les collecteurs.

Au réservoir Seine, les flux particulaires sont de l'ordre de 1600 à 3800 tonnes pour un remplissage annuel de 
Tableau IV. - Ordres de grandeur des flux de matière sèche, de carbone organique particulaire, de l'azote Kjeldahl particulaire, et de phosphore total particulaire, estimés pour un remplissage complet (Seine: $205 \mathrm{Mm}^{3}$, Aube $170 \mathrm{Mm}^{3}$ ).

Table IV. - Orders of magnitude of dry matter, particulate organic carbon, particulate Kjeldahl nitrogen and particulate total phophorus inflows. Estimates done for a whole filling up (Seine: $205 \mathrm{Mm}^{3}$, Aube $170 \mathrm{Mm}^{3}$ ).

\begin{tabular}{|c|c|c|c|c|}
\hline & \multicolumn{2}{|c|}{ SEINE } & \multicolumn{2}{c|}{ AUBE } \\
\cline { 2 - 5 } & 1992 & 1993 & 1992 & 1993 \\
\hline matière sèche (tonnes/an) & 3800 & 1600 & 760 & 756 \\
\hline $\begin{array}{c}\text { carbone organique } \\
\text { particulaire (tonnes/an) }\end{array}$ & $174,8 \pm 34,2$ & $96,3 \pm 76,8$ & $41,0 \pm 9,1$ & $40,3 \pm 17,2$ \\
\hline $\begin{array}{c}\text { azote Kjeldahl particulaire } \\
\text { (tonnes/an) }\end{array}$ & $19,76 \pm 3,80$ & $10,08 \pm 4,16$ & $4,64 \pm 2,13$ & $5,30 \pm 2,72$ \\
\hline $\begin{array}{c}\text { phosphore total particulaire } \\
\text { (tonnes/an) }\end{array}$ & $4,90 \pm 4,18$ & $5,63 \pm 4,21$ & $0,96 \pm 0,34$ & $1,98 \pm 1,31$ \\
\hline
\end{tabular}

$205 \mathrm{Mm}^{3}$, ce qui correspond à une concentration moyenne de 9 à $18 \mathrm{mg} / \mathrm{l}$. Les variations interannuelles apparaissent importantes pour les flux de carbone organique particulaire (COP1992 : 174,8 tonnes/an, COP1993: 96,3 tonnes/an) et pour l'azote Kjeldahl particulaire (Nk1992: 19,76 tonnes/an, Nk1993: 10,08 tonnes/an). Les flux de phosphore total semblent plus stables pour les deux années et sont de l'ordre de 4,90 et 5,63 tonnes/an.

$\mathrm{Au}$ réservoir Aube, les flux de matière sèche et des constituants analysés montrent une variabilité interannuelle plus modérée et les estimations pour 1992 et 1993 se situent dans les mêmes ordres de grandeurs (M.S. : 760 - 756 tonnes/an, COP : $41,0-40,3, \mathrm{Nk}: 4,64-5,90$ tonnes/an, Pt : $0,96-1,31$ tonnes/an).

Bien que les estimations soient formulées avec des amplitudes assez larges, il apparaît des valeurs sensiblement plus élevées sur le réservoir Seine que sur le réservoir Aube. On peut vraisemblablement voir dans ce résultat l'incidence d'un ouvrage de dessablement existant en tête du canal d'amenée du réservoir Aube.

La mise en mouvement et le transport des particules solides par le ruissellement, l'érosion des berges et la remise en suspension des sédiments déposés dans le lit des rivières s'effectuent principalement en période de crue (Meybeck 1985). On observe généralement que le «débit solide» maximum précède la période de plus fort débit de la rivière, ce qui se traduit à débit égal par une nette différence de la concentration en MES entre la crue et la décrue. Dans un même ordre d'idée, sur un cycle hydrologique, les premières crues apparaissent les plus turbides (Walling 1988). 
Entre les mois de novembre et décembre, à l'ouverture des canaux, la matière organique sur le canal Aube est essentiellement constituée par les algues filamenteuses ayant proliférées durant l'été. Sur le canal Seine,

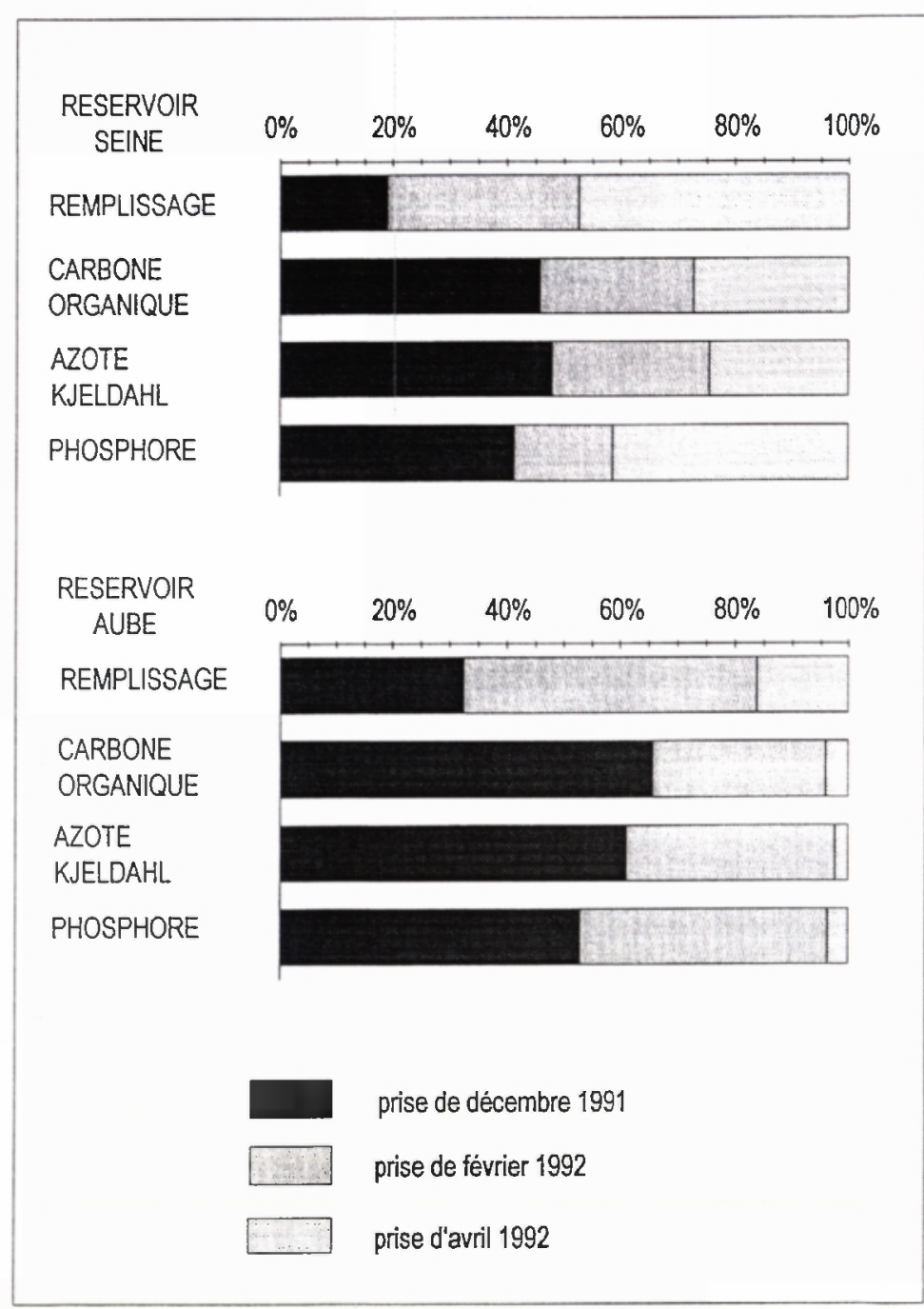

Fig. 8. - Effel de la période de remplissage sur les flux du carbone organique, de l'azote KjeldahJ, et du phosphore. Les flux hydrauliques et particulaires sont exprimés en pour cent du total de la période d'étude.

Fig. 8. - Effect of the filling up period on constituents inflow (organic carbon, Kjeldahl nitrogen, phosphorus). Water discharge and constituents inflow are expressed in per cent of the whole period. 
ce sont surtout des macrophytes Ranunculus sp. qui constitue l'essentiel de la biomasse. Les éléments organo-détritiques (feuilles mortes, débris végétaux divers) deviennent dominants avec l'avancée de la saison hivernale.

A la figure 8 , on apprécie l'impact de la période de prise sur l'entrée des constituants. Sur le canal Aube on estime que 60 à $70 \%$ des entrées s'effectuent au cours de la première période de prise qui représente environ $40 \%$ du débit cumulé. Cette observation est plus nettement mise en évidence au réservoir Seine où 50 à $55 \%$ des entrées correspondent à $25 \%$ du volume de remplissage.

\section{CONCLUSIONS}

Les avantages de la méthode de prélèvement en continu par la mise en place de collecteurs, sont essentiellement liés à la quantité de matière récoltée, qui permet d'effectuer toutes les analyses sans nécessiter de techniques analytiques spécifiques, et à l'autonomie d'un matériel peu onéreux, qui ouvre la possibilité d'obtenir des résultats simultanés sur plusieurs stations très éloignées.

Nos investigations menées sur les canaux d'amenée Aube et Seine au cours des années 1992 et 1993 montrent la concordance des résultats obtenus par les collecteurs et sur les sédiments ou les matières en suspension. L'établissement des courbes de calibration par le calcul des constantes de régression entre les trois compo- santes (débits, flux particulaires, flux captés par les collecteurs) s'avère envisageable et constitue l'objectif des expérimentations en cours. Elles devraient permettre à terme, une approximation fiable des apports particulaires dérivés annuellement dans les réservoirs, au seul vu des débits de prise par ailleurs contrôlés et connus.

Les travaux actuels se poursuivent sur les canaux de restitution et incluent le réservoir Marne. L'étude de la fraction particulaire s'intègre dans un plus large programme de "suivi écologique" des barrages-réservoirs dont l'Institution a charge de gestion.

\section{REMERCIEMENTS}

Le financement du projet est assuré par l'Institution Interdépartementale des Barrages-Réservoirs du Bassin de la Seine, nous remercions Monsieur Rizzoli (I.I.B.R.B.S) et Monsieur Jampi (I.I.B.R.B.S.), pour la confiance et le soutien permanent qu'ils nous ont accordé. Nous remercions Monsieur Mouchel (C.E.R.G.R.E.N.E) et Monsieur Meybeck (Université Paris 6), pour leurs suggestions lors de la conception des collecteurs et la mise en place du protocole. Nous remercions $\mathrm{M}$. Remy et $\mathrm{M}$. Botella du Laboratoire de Chimie des Eaux de Besançon qui ont effectué les analyses des sédiments et des suspensions.

\section{AUTEURS CITÉS}

Bapst A., 1987. Le lac de Neuchâtel : physico-chimie et turbidimétrie des eaux. Concentration, minéralogie et granulométrie des particules en suspension. Th. Univ. Neuchâtel. 101 p. 
Bloesch J. \& Evans R.D., 1982. Lead-210 dating of sediments compared with accumulation rates estimated by natural markers and measured with sediment traps. Hydrobiologia, 92, 579-586.

Dussart B., 1966. Limnologie : l'étude des eaux continentales. Gauthier Villars (éd.), Paris, $675 \mathrm{p}$.

Ertel J.R., Hedges J.I., Devol A.H., Richey J.E. \& De Nazaré Goés Ribeiro M., 1986. Dissolved humic substances of the Amazon River system. Limnol. Oceanogr. 31 (4) : 739-754.

Fattorelli S., Lenzi M., Marchi L. \& Keller H.M., 1988. An experimental station for the automatic recording of water and sediment discharge in a small alpine watershed. Hydrological Sciences, 33, (6) : 607-617

Favarget P.-Y. \& Vernet J.P., 1991. Transport d'éléments-trace par les particules en suspension en rivière : comparaison de qualques méthodes de quantification. In Vernet J.P. (Ed.) : Hommage à F.-A. Forel. 71-74. CILEF 3, Morges, mai 1991.

Fisher J.S., Pickral J. \& Odum W.E., 1979. Organic detritus particles: Initiation of motion criteria. Limnol. Oceanogr., 24 (3) : 529-532.

Golterman H.L., 1973. Natural phosphates sources in relation to phosphate budgets: a contribution to the understanding of eutrophication. Water Research, $7: 3-17$.

Golterman H.L., Sly P.G. \& Thomas R.L., 1983. Study of the relationship between water quality and sediment transport. UNESCO (ed.), Paris, $231 \mathrm{p}$.

Hakanson L. \& Jansson M., 1983. Principles of lakes Sedimentology. SpringerVerlag, Berlin, Heidelberg, New-York, Tokyo. 309 p.
Hedges J.I., Clark W.A., Quay P.D., Richey J.E., Devol A.H. \& De Santos U., 1986. Compositions and fluxes of particulate organic material in the Amazon River. Limnol. Oceanogr., 31, (4) : 717-738.

Jaquet J. M., 1985. Bilan et modèle d'entrée - sorties du phosphore total dans le Léman. Annls. Limnol. 21 (3) : $177-$ 189.

Martin C., 1993. Analyse comparative du seston estival de 9 lacs du jura français. Annis. Limnol., 29 (3-4) : 365-381.

Meybeck M., 1983. Atmospheric inputs and river transport of dissolved substances. In: Dissolveed Loads of Rivers and Surface Water Quantity/Quality Relationships. Proceed. Hamburg Symp., august 1983. IAHS (ed.), $141: 173-191$.

Meybeck M., 1985. Variabilité dans le temps de la composition chimique des rivières et de leurs transports en solution et en suspension. Revue Française des Sciences de l'Eau, 4 : 93-121.

Rodier J., 1984. L'analyse de l'eau. Eaux naturelles - Eaux résiduaires - Eaux de mer. $7^{e}$ éd., Dunod (éd.), Paris, $1365 \mathrm{p}$.

Stabel H.-H. \& Geiger M., 1985. phosphorus adsorption to riverine suspended matter. Implication for the P-budget of lake Constance. Water Resarch, 19, (11) : 1347-1352.

Van Put A., Grieken R.V., Wilken R.-D., \& Hudec B., 1994. Geochemical characterization of suspended matter and sediment samples from the Elbe River by EPXMA. Water Res., 28, (3) : 643655.

Walling D.E., 1988. Erosion and sediment yield research. Some recent perspective. Journal of Hydrology, $100: 113-$ 141. 